\title{
Field dependence of the Josephson current and Fiske resonances in specially shaped Josephson junctions
}

\author{
J.G. Gijsbertsen ${ }^{\text {a, }}$, E.P. Houwman ${ }^{\text {a }}$, B.B.G. Klopman ${ }^{\text {a }}$, J. Flokstra ${ }^{\text {a }}$, H. Rogalla ${ }^{\text {a }}$, \\ D. Quenter ${ }^{b, 1}$, S. Lemke ${ }^{b}$ \\ ${ }^{a}$ Department of Applied Physics, Low Temperature Division, University of Twente, PO. Box 217, 7500 AE Enschede, The Netherlands \\ ${ }^{\mathrm{b}}$ Lehrstuhl Experimentalphysik II, University of Tübingen, Morgenstelle 14, D-72076 Tübingen, Germany
}

Received 25 February 1995; revised manuscript received 11 May 1995

\begin{abstract}
We investigated the magnetic-field dependence of the Josephson current and Fiske resonances in specially shaped Josephson junctions. In order to be able to use junctions for high-resolution X-ray spectroscopy, a very good suppression of the sidelobes of both the Josephson current and the Fiske resonances must be achieved.

In a theoretical argument we show that a properly chosen junction shape leads to the sidelobe suppression of both the critical current and Fiske resonance amplitudes.

The Josephson current and Fiske resonance amplitudes were measured as a function of the magnetic field, for junctions fabricated in $\mathrm{Nb} / \mathrm{Al}$ technology. As expected, a very good sidelobe suppression was obtained for quartic-shaped junctions. For junctions with anodized structures within the tunneling area, the shape of the internal structures is reflected in the field dependence of both the Josephson current and the Fiske resonances. Finally, Fiske modes in these junctions have been imaged with low-temperature scanning electron microscopy, and we conclude that a quartic junction can be approximated by a rectangle, to describe the lower-order Fiske modes, whereas the high-order modes are specific to the exact shape of the junction.
\end{abstract}

\section{Introduction}

When a junction is used to detect $\mathrm{X}$-ray photons or other high-energetic particles, it is most often current biased in the subgap regime [1]. The unwanted switching to either the Josephson zero-voltage state, or to a Fiske resonance, hampers the stable operation as a particle detector. The Fiske resonances

\footnotetext{
* Corresponding author.

${ }^{1}$ Present address: Physikalisch-Technische Bundesanstalt, Bundesallee 100, D-38116 Braunschweig, Germany.
}

are observed for large junctions, which are desired for their large detector area, and appear as constant voltage steps in the $I-V$ curve, if a magnetic field is applied. A sufficiently large magnetic field suppresses both the Josephson current and the Fiske steps to the subgap level. Large fields on the other hand, cause more flux lines to be trapped in the $\mathrm{X}$-ray absorber (normally one of the junction electrodes), leading to an increase of the quasiparticle loss by trapping [2]. We are therefore interested in junctions in which the suppression of both the Josephson current and the Fiske steps is obtained for fields that are as small as possible. 
It is well known that the dependence of the Josephson current on the magnetic field can be easily manipulated by changing the junction shape. It has been demonstrated, both theoretically [3] and experimentally [4], that $I_{c}(B)$ curves with very well suppressed sidelobes are obtained for junctions with special geometries. Especially a quartic-shaped junction gives an extremely good suppression of the sidelobes.

Fiske resonances in a quartic junction are also suppressed to the subgap level by a relatively small field. Preliminary results indicated that the insertion of anodized structures inside the tunneling area, designed to reduce the resonances, do not lead to an extra suppression of Fiske steps [5], indicating that a more thorough study to understand the effect of such structures on the Fiske resonances was necessary.

Next to magnetic-field dependent measurements, spatially resolved information about the Fiske resonances can be obtained by imaging the mode patterns of the resonant magnetic field with low-temperature scanning electron microscopy (LTSEM) [6].

A theoretical argument that shows how the fielddependent behavior of the Josephson current and the Fiske resonances, in junctions with these special geometries, is influenced by the junction shape, is given in Section 2. Measurements of the critical current and a number of Fiske resonance peak amplitudes as a function of the magnetic field are presented and discussed in Sections 3-5. For the experiment we used quartic junctions with and without anodized structures within the barrier area. Finally, images of the Fiske resonance modes obtained with the LTSEM are discussed in Section 6.

\section{Theory}

In the following, we will investigate the role of the junction shape in the magnetic-field dependence of the Josephson current and the Fiske resonances. The spatial shape of the boundary of a symmetrically shaped junction is described by the positive and even function $f(x)$ for $y>0$, and by $-f(x)$ for $y<0$, respectively. The origin of the coordinate system is located at the center of the junction. The dimensions in the $x$ and $y$ direction are respectively given by $L$ and $W$. Fig. 1 shows the outline of a junction defined

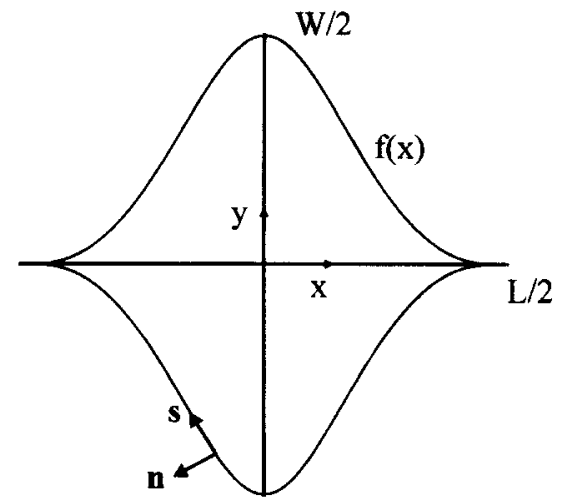

Fig. 1. Shape of a quartic junction. For $|x| \leq L / 2$ the even and positive function $f(x)$ describes the boundary of the junction barrier for $y>0$, and $-f(x)$ for $y<0$. For $|x|>L / 2$ we define $f(x)=0$. The unit vectors shown are the outward pointing normal $n$, and the tangential unit vector $s$, respectively. Both vectors are in the plane of the junciion.

by the quartic polynomial $f(x)=y_{0}\left(1-6 \xi^{2}+\right.$ $\left.8|\xi|^{3}-3 \xi^{4}\right)$ for $|x| \leq L / 2$, and $f(x)=0$ for $|x|>L / 2$, where $y_{0}=W / 2$ and $\xi=x /(L / 2)$. In the following, this junction shape will be referred to as quartic.

Assuming a homogeneous tunneling barrier with a maximum critical current density $J_{1}$, the Josephson current or the DC current due to a Fiske resonance follows from the time-averaged spatial integral of the local Josephson current density $J_{1} \varphi(x, y, t)$ over the area of the tunneling barrier:

$$
\begin{aligned}
I_{\mathrm{DC}}= & J_{1} \lim _{T \rightarrow \infty} \frac{1}{T} \\
& \times \int_{0}^{T} \int_{-L / 2}^{L / 2} \int_{-f(x)}^{f(x)} \sin \varphi(x, y, t) \mathrm{d} y \mathrm{~d} x \mathrm{~d} t .
\end{aligned}
$$

\subsection{Josephson current}

The influence of the self-field can be neglected if the junction dimensions are smaller than about twice the Josephson penetration length $\lambda_{\mathrm{J}}=\left(\Phi_{0} /\right.$ $\left.2 \pi \mu_{0} d J_{1}\right)^{1 / 2}$. The flux quantum $\Phi_{0}$ and the magnetic permeability in vacuum $\mu_{0}$ are physical constants, and the magnetic thickness is $d=2 \lambda_{\mathrm{L}}+t$, wherein $\lambda_{\mathrm{L}}$ is the London penetration depth, assumed equal for both electrodes, and $t$ is the barrier 
thickness. The local phase difference between the electrodes $\varphi(x, y, t)=k_{y} x+\varphi_{0}$ is entirely determined by the external magnetic field $B_{y}$, that is applied in the $y$ direction and is represented by the wave vector $k_{y}=2 \pi B_{y} d / \Phi_{0}$. The dependence of the total critical current on the applied magnetic field $I_{c}(B)$ may now be written as $[3,7]$ :

$I_{c}(B)=2 J_{1}\left|\int_{-L / 2}^{L / 2} f(x) \cos \left(k_{y} x\right) \mathrm{d} x\right|$,

which is proportional to the absolute value of the cosine Fourier transform of the junction shape function $f(x)$. The phase constant $\varphi_{0}$ is chosen to maximize the critical current. Peterson [3] calculated $I_{\mathrm{c}}(B)$ dependences with envelope functions falling off as $1 / B, 1 / B^{2}, 1 / B^{3}$, and $1 / B^{4}$, for square, diamond, " $1+$ cosine" and quartic-shaped junctions, respectively.
If within the tunneling area a certain area, described by the function $a(x)$ in the same way as the outer shape is described by $f(x)$, does not carry any Josephson current, e.g. because it is anodized, $f(x)$ is replaced by $f(x)-a(x)$ and we write

$$
I_{c}(B)=2 J_{1}\left|\int_{-L / 2}^{L / 2}(f(x)-a(x)) \cos \left(k_{y} x\right) \mathrm{d} x\right| \text {. }
$$

The first photograph in Fig. 2 shows an example of such a junction with a rectangularly shaped internal structure. For a centered rectangle with width $U$ and height $V, a(x)$ is defined as

$a(x)= \begin{cases}V / 2, & \text { for }|x| \leqslant U / 2, \\ 0, & \text { for }|x|>U / 2 .\end{cases}$

The original $I_{\mathrm{c}}(B)$ dependence, given by $f(x)$, can be regained by replacing the outer shape $f(x)$ by
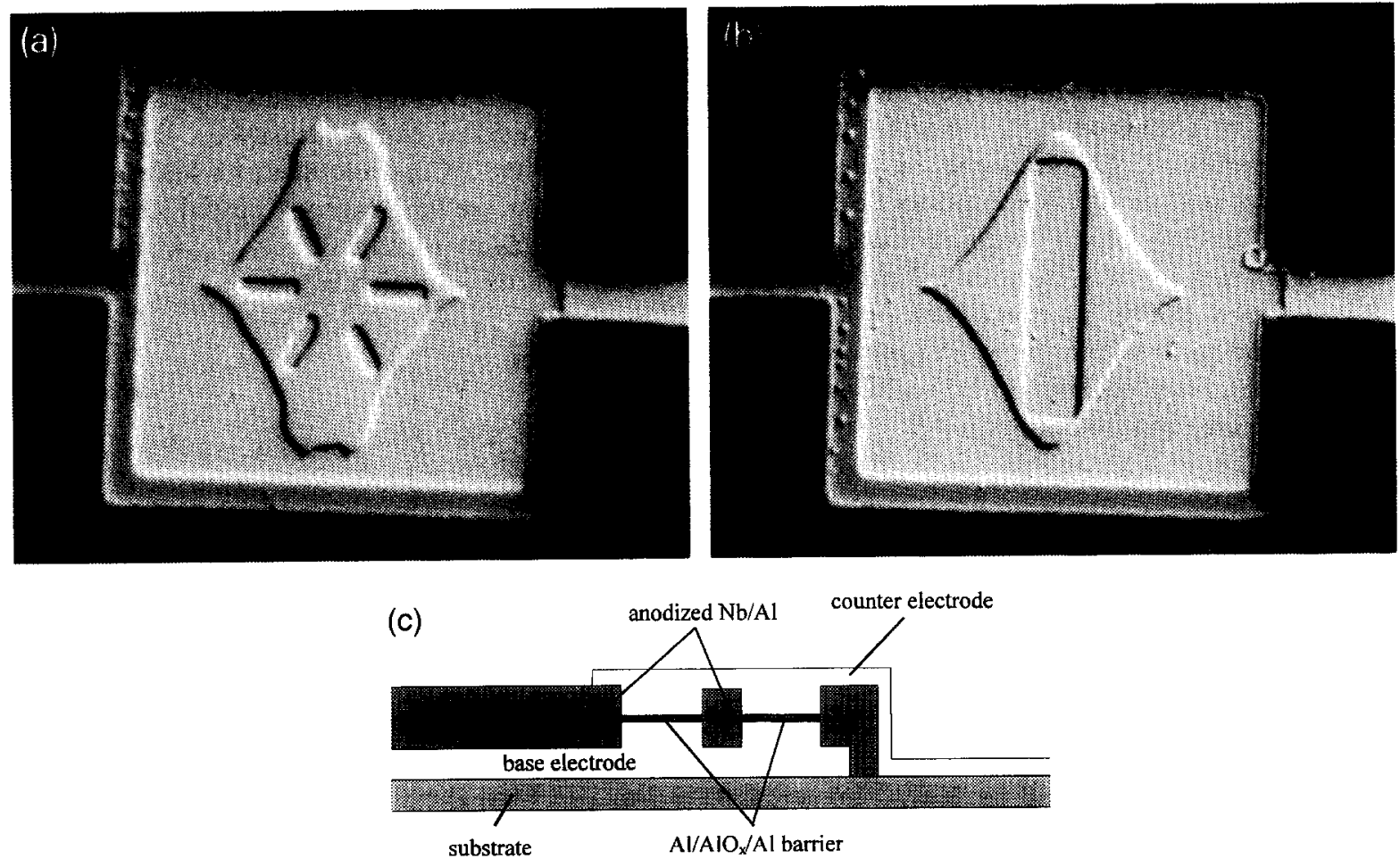

Fig. 2. Photographs of the "quartic with bar" junction (2), and the "quartic with cross" junction (3) with outer dimensions $L=W=54$ $\mu \mathrm{m}$, and a schematic cross-section of the quartic junction with a vertical bar. The cross-section is taken along the current leads, perpendicular to the bar. 
$f(x)+a(x)$ to compensate for the effect of the internal structures.

\subsection{Fiske resonances}

Fiske resonances arise from the non-linear interaction between the AC Josephson current and the two-dimensional electromagnetic cavity modes in the junction barrier. The local phase difference $\varphi(x, y, t)$ between the superconducting electrodes, that gives the total DC current in Eq. (1), can be found as the solution of the two-dimensional perturbed sine-Gordon equation (PSGE, see for instance Refs. [7] and [8]:

$$
\begin{aligned}
\varphi_{x x}+\varphi_{y y}-\frac{1}{\bar{c}^{2}} \varphi_{t t}= & \frac{1}{\lambda_{\mathrm{J}}^{2}} \sin \varphi+\alpha \varphi_{t} \\
& -\beta\left(\varphi_{x x t}+\varphi_{y y t}\right),
\end{aligned}
$$

where $\bar{c}=c(t / \varepsilon, d)^{1 / 2}$ is the Swihart velocity for a barrier with a relative dielectric constant $\varepsilon_{\mathrm{r}}$. The subscripts denote partial derivatives. The damping due to the quasiparticle tunneling current, described by the shunt conductance per unit area $G$, is given by $\alpha=G \mu_{0} d$. The damping caused by the surface resistance $R_{\mathrm{s}}$ is represented by $\beta=\mu_{0} d / R_{\mathrm{s}}$. Neglecting both the influence of the field induced by the bias current, which is allowed for small junctions, and (in the boundary conditions) the effect of the resonant field induced by normal electrons flowing parallel to the barrier, the boundary conditions for junctions of arbitrary shape can be written as

$\varphi_{\mathrm{n}}=\frac{-H_{s}}{J_{1} \lambda_{J}^{2}}$

which is a direct result of the basic relation between the local magnetic field and the phase difference $\varphi$ and the notion that the resonant magnetic field in the tangential direction must be zero. The index $\mathrm{n}$ denotes the derivative along the normal $n$. The magnetic field $H_{\mathrm{s}}$ is taken in the $s$ direction, i.e. along the tangential unit vector $s$ (see Fig. 1). The derivative along the tangential unit vector $\varphi_{s}$ is non-zero, in general. The resulting boundary conditions in the case of a rectangular junction can be found in Ref. [8], and references therein.
To our knowledge, the complex case of Fiske modes in junctions with a special shape, e.g. quartic, has not been investigated theoretically in the literature. A numerical approach to exactly solve the PSGE seems necessary. For junctions with internal structures, the situation is even more complicated. A proper description has to deal with boundary conditions for the boundaries between the barrier area and the internal regions, where the electrodynamics are described by a modification of the PSGE, and these boundary conditions will describe the reflection of the standing waves at these boundaries.

Notwithstanding the fact, that the solution of the PSGE for resonances in a specially shaped junction cannot be easily obtained, it can be demonstrated how the shape function $f(x)$ influences the magnetic-field dependence of the Fiske resonances $I_{\mathrm{F}}(B)$. We will write the phase function $\varphi(x, y, t)=\omega t+$ $k_{\mathrm{y}} x+\varphi_{1}(x, y, t)+\varphi_{0}$, where $\omega$ corresponds to the applied DC voltage, and $k_{y}$ is the wave vector describing the magnetic field, that is applied in the $y$ direction. Under the assumption that the resonance is already somewhat suppressed, it is reasonable to regard $\varphi_{1}(x, y, t)$ as a small perturbation, even in the case of high- $Q$ resonances, and we can write

$$
\begin{aligned}
\sin \varphi & (x, y, t) \\
= & \sin \left(\omega t+k_{y} x+\varphi_{0}\right)+\varphi_{1}(x, y, t) \\
& \times \cos \left(\omega t+k_{y} x+\varphi_{0}\right) .
\end{aligned}
$$

The time average of the first term of Eq. (7) equals zero and has no net contribution to the current in Eq. (1). Hence we focus on the second term. In the following, we will omit the phase constant $\varphi_{0}$, since we average over time. First we expand $\varphi_{1}(x, y, t)$ as a Fourier series for each $(x, t)$ between $-f(x)$ and $f(x)$ :

$$
\begin{aligned}
\varphi_{1}(x, y, t)= & \sum_{n=0}^{\infty} a_{n}(x, t) \cos \frac{n \pi y}{f(x)} \\
& +\sum_{n=1}^{\infty} b_{n}(x, t) \sin \frac{n \pi y}{f(x)} .
\end{aligned}
$$

The coefficients $a_{n}$ and $b_{n}$ are determined by the boundary condition Eq. (6). After substitution of Eq. (7) and Eq. (8) in Eq. (1), and integrating over $y$, only the term $a_{0}(x, t)$ gives rise to a non-zero 
contribution. The expression for the total current $I_{\mathrm{DC}}$ can now be written as

$$
\begin{aligned}
I_{\mathrm{DC}}= & 2 J_{1} \lim _{T \rightarrow \infty} \frac{1}{T} \int_{0}^{T} \int_{-L / 2}^{L / 2} f(x) a_{0}(x, t) \\
& \times \cos \left(\omega t+k_{y} x\right) \mathrm{d} x \mathrm{~d} t .
\end{aligned}
$$

Now we expand $a_{0}$ as a Fourier series in the interval $[-L / 2, L / 2]$ :

$$
\begin{aligned}
a_{0}(x, t)= & \sum_{m=0}^{\infty} A_{m}(t) \cos \frac{m \pi x}{L / 2} \\
& +\sum_{m=1}^{\infty} B_{m}(t) \sin \frac{m \pi x}{L / 2} .
\end{aligned}
$$

Rewriting the factor $\cos \left(\omega t+k_{y} x\right)$ and omitting integrands that are odd in $x$, we find for Eq. (9)

$$
\begin{aligned}
I_{\mathrm{DC}}= & \sum_{m=0}^{\infty} \overline{A_{m}(t) \cos \omega t}\left(J_{1} \int_{-L / 2}^{L / 2} f(x)\right. \\
& \times \cos \left[\left(k_{y}+\frac{m \pi}{L / 2}\right) x\right] \mathrm{d} x+J_{1} \int_{-L / 2}^{L / 2} f(x) \\
& \left.\times \cos \left[\left(k_{y}-\frac{m \pi}{L / 2}\right) x\right] \mathrm{d} x\right) \\
& +\sum_{m=1}^{\infty} \overline{B_{m}(t) \sin \omega t}\left(J_{1} \int_{-L / 2}^{L / 2} f(x)\right. \\
& \times \cos \left[\left(k_{y}+\frac{m \pi}{L / 2}\right) x\right] \mathrm{d} x-J_{1} \int_{-L / 2}^{L / 2} f(x) \\
& \left.\times \cos \left[\left(k_{y}-\frac{m \pi}{L / 2}\right) x\right] \mathrm{d} x\right),
\end{aligned}
$$

so that the field dependence of the amplitude of the Fiske resonances $I_{\mathrm{F}}(B)$ takes the form

$I_{F}(B)=\sum_{m=-\infty}^{\infty} C_{m} J_{1} \int_{-L / 2}^{L / 2} f(x) \cos \left(k_{y, m}^{\prime} x\right) \mathrm{d} x$,

where

$k_{y, m}^{\prime}=k_{y}+\frac{m \pi}{L / 2}$,

which is a superposition of cosine Fourier transforms of the shape function $f(x)$, each term being shifted $\pm 2 m \pi / L$ along the $k$-axis. We have already mentioned, that the shape function $f(x)$ can be chosen so that all terms of Eq. (12) have very well suppressed sidelobes as a function of all $k_{y, m}^{\prime}$. Under the assumption, that terms with a very rapid modulation in $x$ (i.e. large $m$ ) are not significant, we can conclude that not only the $I_{\mathrm{c}}(B)$ patterns, but also the $I_{\mathrm{F}}(B)$ patterns, have suppressed sidelobes.

For junctions with anodized internal parts, with and without shape compensation, arguments analogous to the foregoing argument can be given. The $I_{\mathrm{F}}(B)$ curves will reflect contributions of both the outer shape and the shape of the internal structures. For a quartic-shaped junction with a rectangular internal structure inside and without shape compensation, for instance, we expect a Fraunhofer-like behavior for larger magnetic fields, characterized by lobes in the $I_{\mathrm{F}}(B)$ curves.

\section{Sample preparation}

On two oxidized silicon wafers, $\mathrm{HG \# 4}$ and

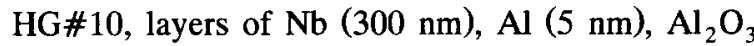
(about $1 \mathrm{~nm}), \mathrm{Al}(3 \mathrm{~nm})$, and $\mathrm{Nb}(30 \mathrm{~nm})$ were deposited and structured with lift-off. We used DC magnetron sputtering to deposit the $\mathrm{Nb}$ and $\mathrm{Al}$ layers and thermal oxidation ( $1 \mathrm{~h}$ at a pressure of 10 and 20

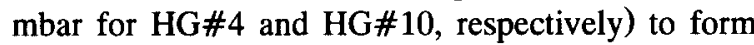
the $\mathrm{Al}_{2} \mathrm{O}_{3}$ barrier.

Subsequently, the junction shapes were defined with the SNAP process [9], with anodization voltages of $47 \mathrm{~V}$ and $57 \mathrm{~V}$, giving $\mathrm{Nb}_{2} \mathrm{O}_{5}$ thicknesses of 108

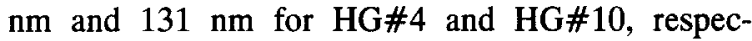
tively. On HG\#4 we patterned square, diamondshaped, " 1 + cosine" shaped, and quartic-shaped junctions, and on HG\#10 we patterned quartic junctions and quartic junctions with various internal structures. The accuracy of structuring was approximately $1 \mu \mathrm{m}$. Finally, a $165 \mathrm{~nm}$ thick counter electrode was deposited, completely covering the barrier area. The base and top electrode show an overlap with respect to the barrier of at least $3 \mu \mathrm{m}$ all around the barrier (see photographs in Fig. 2).

The electrical measurements that are described below were performed on three samples ( $L=W=54$ $\mu \mathrm{m})$ of HG\#10:

(1) a quartic junction without any anodized structure inside, 
(2) a quartic junction with a bar of $8 \times 40 \mu \mathrm{m}^{2}$ (designed value), but no compensated outer shape, (3) a quartic junction with a cross and a compensated outer shape. Photographs of the junctions (2) and (3) are shown in Fig. 2, along with a schematic view of the cross-section of the junction with a vertical bar. The cross-section is taken along the current leads, i.e. perpendicular to the bar. The LTSEM measurements were performed on a quartic-shaped junction $(L=W=104 \mu \mathrm{m})$ of HG\#4.

\section{Field dependence of the Josephson current}

\subsection{Experimental}

The samples were measured in a magnetically shielded environment. The magnetic field was applied in the plane of the junction and perpendicular to the current leads, in order to prevent its distortion. The $I_{\mathrm{c}}(B)$ curves were obtained computer-controlled by continuously traversing the $I-V$ curve and measuring the junction current and the magnetic field, when the junction switches to the voltage state. The measurement temperature was $4.2 \mathrm{~K}$.

The measurement of the critical current versus the magnetic field for a $20 \times 20 \mu \mathrm{m}^{2}$ junction on HG\#10 yielded a London penetration depth $\lambda_{\mathrm{L}}=81$ $\mathrm{nm}$. In the following we will assume that all borders between the anodized and the non-anodized parts have shifted equal distances $\Delta L=0.75 \mu \mathrm{m}$ into the direction of the non-anodized parts, which follows from the results shown below. This effect changes the effective junction areas. The real dimensions of the $20 \times 20 \mu \mathrm{m}^{2}$ junction are $18.5 \times 18.5 \mu \mathrm{m}^{2}$, corresponding to a maximum critical current density $J_{1}=158 \mathrm{~A} / \mathrm{cm}^{2}$, and a Josephson penetration length $\lambda_{\mathrm{J}}=32 \mu \mathrm{m}$. The current density of this junction can be considered homogeneous (small junction). The maximum critical current in the investigated specially shaped junctions is expected to be somewhat suppressed by the self-field effect, since their dimensions are comparable to $\lambda_{\mathrm{J}}$.

\subsection{Quartic}

The $I_{c}(B)$ pattern of the quartic junction (1) is shown in Fig. 3(a). The maximum critical current
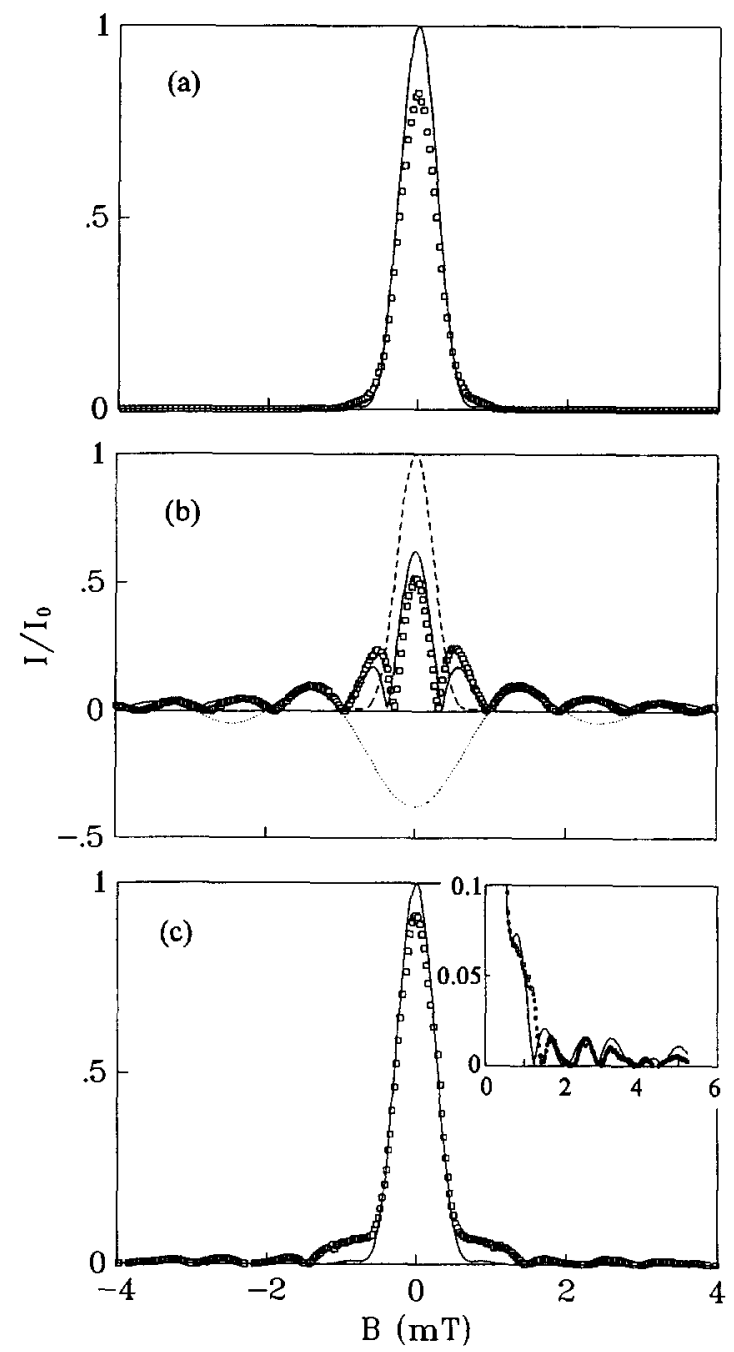

Fig. 3. Experimental $(\square)$ and theoretical (solid line) $I_{c}(B)$ curves of (a) the quartic junction (1), (b) the "quartic with bar" junction (2), and (c) the "quartic with cross" junction (3). In (b) the cosine Fourier transforms of the quartic outer shape (dashed) and the bar (dotted) are also shown. The inset in (c) shows a magnification of the measured data, along with a fit to model the difference shape.

expected for this junction, assuming the homogeneous critical current density $J_{1}=158 \mathrm{~A} / \mathrm{cm}^{2}$, can be calculated from the effective junction area $A_{\mathrm{qu}}=$ $A_{\text {design }}-\Delta A=1166-\Delta L^{*} P \mu \mathrm{m}^{2}$. The barrier perimeter $P \approx 160 \mu \mathrm{m}$ and $\Delta L=0.75 \mu \mathrm{m}$ give $A_{\mathrm{qu}}=1046 \mu \mathrm{m}^{2}$ and $I_{0}=I_{\mathrm{c}, \mathrm{qu}, \mathrm{th}}(0)=1.65 \mathrm{~mA}$. This value is taken to normalize the current-axis. The difference between the theoretical maximum critical current and the experimental value $I_{\mathrm{c}, q u}(0)=1.37$ 
$\mathrm{mA}$ is explained by the inhomogeneous current distribution caused by the self-field. The figure also shows the theoretical line calculated with Eq. (2). The parameters $L=54 \mu \mathrm{m}$ and $d=162 \mathrm{~nm}$ determine the values for the magnetic field in the theoretical fit. Experiment and theory fit almost perfectly, in accordance with the results obtained for a similar junction [4]. The sidelobes are suppressed very rapidly to below $1 \%$ of the $I_{c}(0)$ value. A more detailed view of the $I_{c}(B)$ pattern at higher field values shows a modulation caused by the rounding of the junction shape due to the fabrication process [4]. The shoulder, absent in the theoretical curve, may be ascribed to the self-field as well.

\subsection{Quartic with bar}

Both the measured and the theoretical $I_{c}(B)$ patterns of the "quartic with bar" junction (2) are shown in Fig. 3(b). The theoretical line gives the absolute value of the sum of the dashed and the dotted lines. These lines give the cosine Fourier transforms of the quartic outer shape function $f(x)$, and the function $a(x)$ describing the vertical bar inside, respectively. It can be clearly observed how the two shapes determine the $I_{c}(B)$ pattern. The first minimum occurs when $I_{\mathrm{c}, \mathrm{qu}}$ and $I_{\mathrm{c}, \mathrm{bar}}$ are equal and compensate for each other. The other minima occur for integer multiples of flux quanta inside the bar, because for fields larger than about $1 \mathrm{mT}$ the bar dominates the $I_{\mathrm{c}}(B)$ curve, showing the Fraunhofer behavior, whereas the contribution of the quartic outer shape is negligible.

We used again the (calculated) maximum critical current of the full quartic shape $I_{0}=I_{\mathrm{c}, \mathrm{qu}, \mathrm{th}}(0)=1.65$ $\mathrm{mA}$ to normalize the current-axis. We calculated the maximum critical current of the bar $I_{\mathrm{c}, \mathrm{bar}}(0)=0.63$ $\mathrm{mA}$ from the area and the maximum homogeneous critical current density. The fairly large discrepancy between the measured $I_{c, q u \text {-bar }}(0)=0.85 \mathrm{~mA}$ and the maximum critical current, expected for the area consisting of the quartic minus the bar $I_{c, q u-b a r, t h}(0)=1.02$ $\mathrm{mA}$ is explained by the self-field effect. We note here, that the $I_{c}(B)$ curves are less influenced by the self-field effect at high fields, since the current in the leads is smaller.

To scale the $x$-axis, we took $L=54 \mu \mathrm{m}$ and $L_{\text {bar }}=9.5 \mu \mathrm{m}$. It appeared that the minima of the
Fraunhofer-like pattern due to the bar fitted well for a 1.35 times larger effective magnetic wave vector $k$, which arises from the larger magnetic thickness $d_{\mathrm{Nb}_{2} \mathrm{O}_{5}}=293 \mathrm{~nm}$ of the anodized areas, which is larger than the magnetic thickness of the barrier $d$. The result is that the anodized areas tend to focus (attract) the flux lines, because this is energetically favorable, although not to the extent that the local magnetic field between the superconducting electrodes equals the applied field. In the latter case the wave vector would be $d_{\mathrm{Nb}_{2} \mathrm{O}_{5}} / d \approx 1.8$ times larger. This "flux-focusing factor" is determined by both the total geometry of the junction, and the magnitude of the applied field, and has upper and lower bounds of 1.8 and 1 for all field values, respectively.

We see that a very good correspondence between the measured data and the theory is obtained, if a shift of the anodization border, the self-field, and the flux-focusing effect of the anodized areas are taken into account. Only the main lobe and the first sidelobes are somewhat smaller than predicted.

\subsection{Quartic with cross}

In Fig. 3(c) the measured and the theoretical $I_{\mathrm{c}}(B)$ curves are shown for the "quartic with cross" junction (3), normalized to $I_{0}=I_{\mathrm{c}, q u+\mathrm{cr}, \mathrm{th}}(0)=1.51$ $\mathrm{mA}$. The area $A_{\mathrm{qu}+\mathrm{cr}}=960 \mu \mathrm{m}^{2}$ is calculated by taking into account the shift of both the perimeter and the internal structures. The experimental value $I_{c, q u+c r}(0)=1.38 \mathrm{~mA}$ is significantly lower, which we ascribe to the influence of the self-field. Because the outer shape compensates for the internal structures, the theoretical curve equals the one for the quartic junction. The measured $I_{c}(B)$ dependence shows a relatively good suppression of the sidelobes, although not as good as for the plain quartic junction. This can be explained by a deviation from the designed sizes due to structuring inaccuracy. If the anodized internal part differs in size from the design, the compensation will be imperfect, which can be modelled by the superposition of a "difference shape" for the calculation of the theoretical line. The inset shows an enlargement of the measured $I_{\mathrm{c}}(B)$ pattern of the compensated quartic with a cross inside, and the theoretical pattern of a quartic outer shape, superposed by two horizontal bars of each 6.4 $\mu \mathrm{m}$ wide and $7 \mu \mathrm{m}$ high. The centers of the bars are 


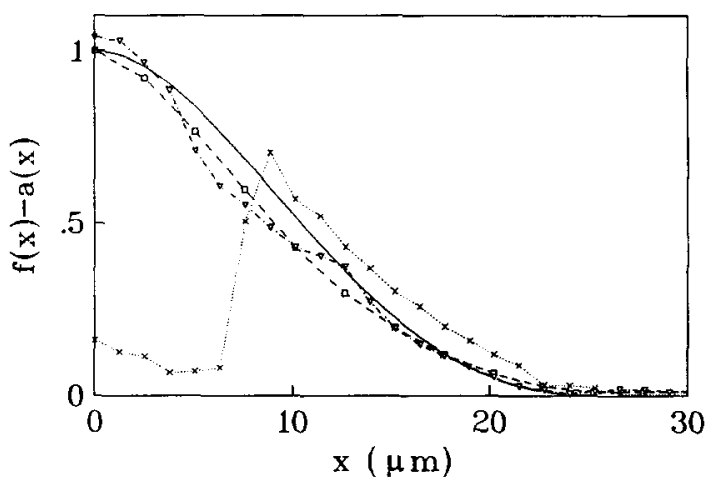

Fig. 4. Fast Fourier transforms (FFT) of the $I_{\mathrm{c}}(B)$ data shown in Fig. 3 and the ideal quartic shape function $f(x) / f(0)=1-6 \xi^{2}$ $+8|\xi|^{3}-3 \xi^{4}$, using $L=54 \mu \mathrm{m}$ (solid line). Quartic junction (1): " $\square$ " + dashed line; "quartic with bar" junction (2): " $X$ " + dotted line; " quartic with cross" junction (3): " $\nabla$ "' + dash-dotted line.

spaced by $15.3 \mu \mathrm{m}$. These numbers are in agreement with the place where one may expect deviations from the cross-structure (see Fig. 2). The corresponding $I_{\mathrm{c}}(B)$ pattern is in good agreement with the measurement. The total area of the modelled "difference structure" corresponded with the amplitude of the theoretical curve. Taking into account the total length of the perimeters of the rectangles forming the star, it is estimated that the borders of the anodized structures have shifted about $0.75 \mu \mathrm{m}$.

\subsection{Fourier transform}

The inverse procedure is to directly Fourier transform the measured $I_{\mathrm{c}}(B)$ data to give a "measured" junction shape function $f(x)$. Fourier transforming should be done after flipping the $I_{\mathrm{c}}(B)$ data of the appropriate lobes over the $x$-axis, to regain the phase information that was lost by the fact that the $I_{c}(B)$ curves display the absolute value of the sum of cosine Fourier transforms. The result of the fast Fourier transform (FFT) algorithm on the experimental $I_{c}(B)$ data is shown in Fig. 4 for the data of the three junctions, along with the ideal quartic-shape function $f(x) / f(0)=1-6 \xi^{2}+8|\xi|^{3}-3 \xi^{4}$, with $L=54 \mu \mathrm{m}$. The spacing of the data on the $x$-axis is determined by the largest magnetic field ( $k$ value). The maximum value $f(0)$ of the data of the quartic junction was taken to normalize the $y$-axis.
The measured shape of the quartic junction deviates only slightly from the exact quartic function. The measured shape function of the quartic with bar clearly shows the vertical bar as a sudden drop for $x<7 \mu \mathrm{m}$. The rounding of the top of the outer shape (near $x=0$ ) is also visible in the measured shape function. The apparent larger width of the bar arises from the flux-focusing effect. For $x>7 \mu \mathrm{m}$, where the measured shape should correspond to the ideal shape, the junction seems to oversize the ideal quartic shape. The "quartic with cross" and compensated outer shape corresponds quite well with the theoretical shape function. The measured shape function is smaller than the ideal shape function for $4<x<10 \mu \mathrm{m}$, due to a shift of the anodized internal structures, and we find a confirmation for modelling this with a double bar as explained in the previous section.

\section{Field dependence of the Fiske resonances}

\subsection{Experimental}

The measurement of the field dependence of the Fiske resonance peak heights $I_{\mathrm{F}}(B)$ was more elaborate. A current source with an adjustable offset was used to sweep through the $I-V$ curve. The minimum value of the current range corresponds to biasing at the foot of the investigated current peak. In this way we were able to measure resonance peaks with a smaller amplitude than a peak at a lower voltage (normally one would switch over the smaller peak). An additional feature was the use of a bias circuit, consisting of a resistor shunting the series combination of the sample and the resistor that monitors the junction current. The load line, i.e. the line along which the junction switches in the $I-V$ curve, has a downward slope, giving a stabler biasing. The dependence of the current peak heights on the magnetic field could even be measured for very small peaks. During the measurement, the magnetic field was applied in the $y$ direction, and the temperature was $4.2 \mathrm{~K}$, unless mentioned otherwise.

\subsection{Quartic}

For the quartic junction (1) we observed Fiske resonances at a number of voltages, that are listed in 
Table 1. Here we also listed the theoretical resonance voltages $V_{\text {th }}=(h \bar{c} / 4 e L)\left(n^{2}+m^{2}\right)^{1 / 2}$ for the $(n, m)$ modes in a $41 \times 41 \mu \mathrm{m}^{2}$ square junction with the magnetic field applied at an angle of $45^{\circ}$ with respect to the $x$-axis, but in the plane of the barrier. We calculated the Swihart velocity $\bar{c}=0.036 c$ from the resonance voltage $(0.56 \mathrm{mV})$ of the first Fiske step of a $20 \times 20 \mu \mathrm{m}^{2}$ square junction on the same wafer HG\#10

The resonance voltages are inversely proportional to the junction size $L$, and tend to be more closely spaced for higher-order modes, i.e. for larger $(n, m)$ values. Due to an increase of the London penetration depth for higher-order modes, leading to a decrease of the Swihart velocity, this effect is even more pronounced [10].

The lower-order modes measured for the quartic junction can be regarded as quasi-rectangular, since the shape of the quartic junction does not deviate much from a $45^{\circ}$ rotated square, in comparison to the resonance wavelength. The resonance measured at $0.38 \mathrm{mV}$ can be interpreted as a quasi $(1,1)$ mode. For a square junction at $45^{\circ}$, this mode is dominant. For higher-order modes the resonance voltages are too closely spaced to distinguish for instance a quasi $(3,3)$ mode. Therefore, it is difficult to obtain an unambiguous one-to-one correspondence for all resonances in Table 1.

The magnetic-field dependence of the amplitudes of the major Fiske resonances $I_{\mathrm{F}}(B)$, i.e. the measured current minus the subgap current, for the quar-

Table 1

Resonance voltages measured for the quartic junction, idem for the quartic with cross, and the theoretical resonance voltages of $(n, m)$ modes in a square $41 \times 41 \mu \mathrm{m}^{2}$ junction with a Swihart velocity $\bar{c}=0.036 c$ (i.e. the same as the quartic junction (1) has). Resonances at higher voltages are not listed in this table

\begin{tabular}{rrll}
\hline $\begin{array}{l}V_{\mathrm{qu}} \\
(\mathrm{mV})\end{array}$ & $\begin{array}{l}V_{\mathrm{qc}} \\
(\mathrm{mV})\end{array}$ & Mode $(n, m)$ & $\begin{array}{l}V_{\mathrm{th}} \\
(\mathrm{mV})\end{array}$ \\
\hline 0.19 & 0.21 & $(1,0)$ & 0.27 \\
F1 0.38 & F1 0.40 & $(1,1)$ & 0.39 \\
F2 0.54 & F2 0.49 & $(2,0)$ & 0.55 \\
F3 0.62 & F3 0.62 & $(2,1)$ & 0.61 \\
F4 0.74 & 0.72 & $(2,2)$ & 0.77 \\
0.82 & F4 0.79 & $(3,0)$ & 0.82 \\
F5 0.88 & F5 0.88 & $(3,1)$ & 0.86 \\
more & more & $(3,2)$ & 0.99 \\
& & $(3,3)$ & 1.09 \\
\hline
\end{tabular}
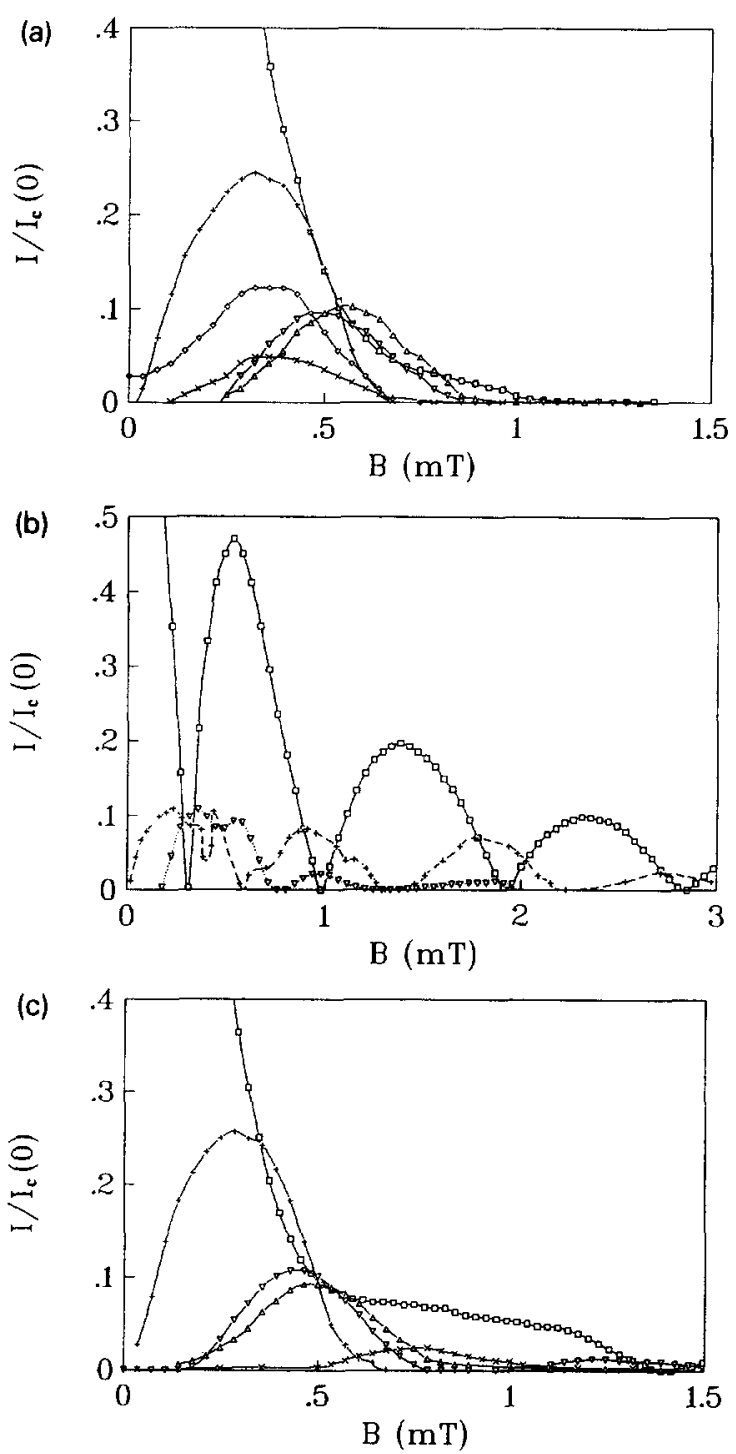

Fig. 5. Experimental $I_{\mathrm{F}}(B)$ curves of (a) the quartic junction (1); (b) the "quartic with bar" junction (2); and (c) the "quartic with cross" junction (3). The lines through the data plots are drawn as a guide for the eye. For (a): F1 at $0.38 \mathrm{mV}(+), F 2$ at $0.54 \mathrm{mV}$ $(\nabla), \mathrm{F} 3$ at $0.62 \mathrm{mV}(\diamond), \mathrm{F} 4$ at $0.74 \mathrm{mV}(\times)$, and F5 at $0.88 \mathrm{mV}$ $(\Delta)$. For (b): $F 1$ at $0.36 \mathrm{mV}(+)$, and $F 2$ at $0.54 \mathrm{mV}(\nabla)$. For (c): F1 at $0.40 \mathrm{mV}(+), F 2$ at $0.49 \mathrm{mV}(\nabla), F 3$ at $0.62 \mathrm{mV}$ (no field dependence), F4 at $0.79 \mathrm{mV}(\times)$, and F5 at $0.88 \mathrm{mV}(\Delta)$. The critical current is indicated by squares $(\square)$ in each graph.

tic shaped junction, is shown in Fig. 5(a). All curves show only one maximum, beyond which the resonance current is rapidly suppressed to the level of the subgap current. At lower temperatures $(1.6 \mathrm{~K})$, the 
same behavior is observed. In contrast, the $I_{\mathrm{F}}(B)$ curves of square $\mathrm{Nb} / \mathrm{Al}$ junctions, with the field applied along one of the junction sides, showed multiple maxima [11], and resembled Fraunhofer patterns, shifted along the field axis.

To model the field dependence of the Fiske resonances in the quartic junction, we approximate the quartic shape by a square junction of $41 \mu \mathrm{m}$, where the magnetic field is applied at an angle of $45^{\circ}$ with respect to the $x$-axis (equivalent to the "diamondshaped junction" in Ref. [4]). We are then able to calculate the amplitude of two-dimensional Fiske resonances in rectangular junctions as a function of the magnetic field, applied at an arbitrary angle in the $x-y$ plane, according to Nerenberg et al. [12]. This model assumes that the perturbation $\varphi_{1}(x, y, t)$ of the superconducting phase $\varphi(x, y, t)$ due to the Fiske modes is small. In our $\mathrm{Nb} / \mathrm{Al}$ junctions high- $Q$ resonances have been observed [11], however, and therefore the small-perturbation condition only holds for larger fields, where the resonance amplitudes are small.

The theoretical field dependence of all resonances is shown in Fig. 6, and roughly shows the same features as our experimental result, shown in Fig. 5(a), namely one lobe and a subsequent suppression. This behavior is observed for all realistic values of

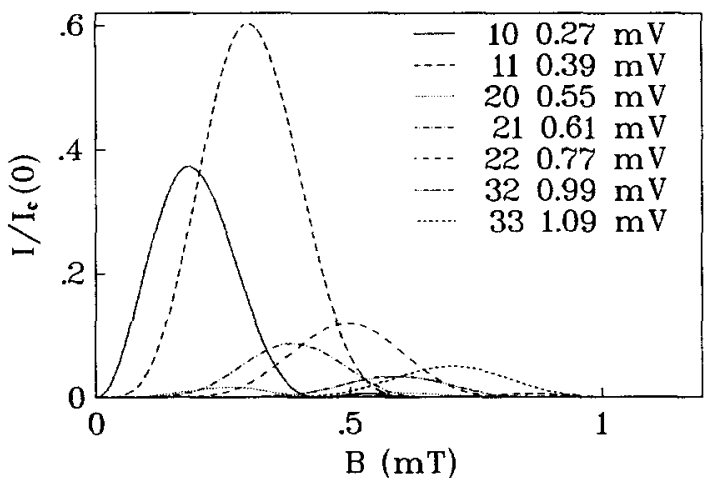

Fig. 6. Theoretical $I_{\mathrm{F}}(B)$ curves for two-dimensional Fiske resonances in a $45^{\circ}$ rotated $41 \times 41 \mu \mathrm{m}^{2}$ square junction, according to Nerenberg et al. [12]. The quality factor $Q$ has been set arbitrarily to the realistic value $25,(1,0)$ mode at $0.27 \mathrm{mV}$ : solid, $(1,1)$ mode at $0.39 \mathrm{mV}$ : dashed, $(2,0)$ mode at $0.55 \mathrm{mV}$ : dotted, $(2,1)$ mode at $0.61 \mathrm{mV}$ : dot-dashed, $(2,2)$ mode at $0.77 \mathrm{mV}$ : mediumdashed, $(3,2)$ mode at $0.99 \mathrm{mV}$ : dot-dot-dashed, $(3,3)$ mode at $1.09 \mathrm{mV}$ : short-dashed. The $(3,0)$ and $(3,1)$ modes are not shown, since they are very small compared to the other modes.
$Q$. Due to the fairly good approximation of the quartic shape by a $45^{\circ}$ rotated square, the Nerenberg model describes the field dependence of the low-order modes in a quartic junction reasonably well.

According to the theoretical argument given in Section 2, the field dependence Fig. 5(a) is expected to show an even better sidelobe suppression than the one shown in Fig. 6. At $4.2 \mathrm{~K}$ the magnitude of the subgap current prohibits a verification of this expected difference. However, also at $1.6 \mathrm{~K}$ we observed no second lobe, and we conclude that any second lobe is much smaller than the subgap current, which is significantly less than the maxima of the second lobes in the model of Fig. 6. Hence we conclude that there is a significantly more rapid sidelobe suppression of Fiske resonances in a quartic-shaped junction than in a rotated square.

\subsection{Quartic with bar}

For the "quartic with bar" junction (2), we found Fiske resonances at a number of voltages, most of them different from those of (1), but similarly spaced. It is difficult to compare the resonance voltages, because the shape of junction (2) differs too much from the rotated square, and therefore we do not list them in Table 1. The $I_{\mathrm{F}}(B)$ curves measured at 1.6 $\mathrm{K}$ for the two most dominant modes are shown in Fig. 5(b). We were not able to measure the $I_{\mathrm{F}}(B)$ curves of the other resonances.

For the resonance $\mathrm{F} 1$ at $0.36 \mathrm{mV}$ not only one, but more lobes are observed. The first idea that comes to mind is that it is caused by a resonance due to a standing wave in the anodized bar. From the normalized Swihart velocities $\bar{c} / c=\left(t / \varepsilon_{\mathrm{r}} d\right)^{1 / 2}=$ 0.12 in the anodized regions $\left(\varepsilon_{\mathrm{r}, \mathrm{Nb}_{2} \mathrm{O}_{5}}=29.4\right.$ [13]), we find that the lowest resonance voltage for a resonance in the bar is $0.96 \mathrm{mV}$, largely exceeding $0.36 \mathrm{mV}$, and we conclude that the bar is not likely to support its own resonant modes, but merely distorts the modes.

The field modulation of $\mathrm{F} 1$ has the same periodicity as the $I_{\mathrm{c}}(B)$ curve, i.e. maxima occur at integer multiples of flux quanta inside the anodized bar. From Eq. (12) we understand that the $I_{\mathrm{F}}(B)$ curve reflects the specific shape function of the whole structure through its cosine Fourier transform. In this case one may expect Fraunhofer-like patterns due to 
the rectangle for larger magnetic fields, where the influence of the quartic outer shape is already damped out.

\subsection{Quartic with cross}

The resonance voltages of the "quartic with cross" junction (3) are shown in Table 1, and are quite similar to those measured for the quartic junction (1). The influence of the internal structures on the resonance voltages seems to be quite small. The $I_{\mathrm{F}}(B)$ curves (Fig. 5(c)) show qualitatively the same behavior as those in Fig. 5(a), although some differences can be observed. The first two resonances (F1 and F2) for junctions (1) and (3), have almost the same field dependence, except for the small second lobe in F2 for junction (3). A fairly strong resonance (F3) was observed at the same voltage as for junction (1), but we were not able to measure the field dependence. The modes F4 show quite different peak amplitudes in both junctions, whereas the modes F5 behave similarly.

It is understandable that the magnetic-field dependence of the resonances is similar to the results obtained for the quartic junction. If the shape compensation works well, all cosine Fourier transforms in Eq. (12) become very small for large fields. In Section 4 it was already shown that for the "quartic with cross" junction (3) the shape compensation does not completely hold, and a "difference shape" has to be accounted for, which is probably responsible for the second lobe of F2.

It is also seen that the $I_{\mathrm{F}}(B)$ curves for resonances in junctions (1) and (3) at lower voltages show a better agreement than those at higher voltages. This can be explained by the fact that the standing waves in the microwave cavity, formed by the combination of the barrier and the anodized internal structures, are not much reflected at the boundary between both regions, as long as the anodized structures are small compared to the wavelength of the resonance (i.e. for low-order modes). Indeed, from the resonance voltages we estimated wavelengths ranging from 20 to $100 \mu \mathrm{m}$ for the resonances in the barrier area. This explains why the influence of the smaller "star", on the mode patterns, and hence on the resonance voltages, is smaller than that of the larger bar, which much more distorts the electromagnetic resonances.

\section{Imaging of Fiske resonances by LTSEM}

In addition to the magnetic-field dependent measurements, images of Fiske resonances in quarticshaped junctions have been obtained by low-temperature scanning electron microscopy (LTSEM). This technique has been extensively described in Ref. [14], and can also be used to obtain two-dimensional images of Fiske resonance modes [6,8]. A summary of the principles is given below.

For imaging, the junction is current biased on a Fiske step in the $I-V$ curve. A voltage image is obtained by monitoring the electron-beam $(25 \mathrm{keV})$ induced change of the junction voltage $\Delta V\left(x_{0}, y_{0}\right)$, as a function of the beam position $\left(x_{0}, y_{0}\right)$ on the junction surface. The lateral resolution is mainly determined by the thermal healing length of the sample, and is on the order of $1 \mu \mathrm{m}$.

The physical interpretation of the obtained image depends on the bias point in the $I-V$ curve and on the mechanisms which cause the beam-induced voltage change. For our purpose the beam power must be carefully adjusted to ensure that the beam acts as a passive probe. The main effect of the electron-beam irradiation is a local increase of the temperature, and hence an increase of the quasiparticle losses ( $\alpha$ term) and the surface losses ( $\beta$ term). Regarding the Josephson junction as an open-ended microwave cavity, the dominant effect is the increase of the surface losses. This causes a reduction of the quality factor $Q$ of the resonance mode in the junction, which is defined as the electromagnetic energy stored in the cavity mode divided by the energy lost per cycle. The detected voltage signal $-\Delta V\left(x_{0}, y_{0}\right)$ is proportional to the beam-induced change of the surface losses $\Delta P_{\mathrm{s}}$. According to basic microwave theory we can write

$\Delta P_{\mathrm{s}}=\frac{1}{2} \Delta \operatorname{Re}\left\{Z_{\mathrm{s}}\right\} \int_{S}\left|\boldsymbol{H}_{\mathrm{tan}}(x, y)\right|^{2} \mathrm{~d} x \mathrm{~d} y$

per electrode, where $\Delta \operatorname{Re}\left\{Z_{\mathrm{s}}\right\}$ denotes the beaminduced change of the real part of the surface impedance $Z_{\mathrm{s}}$ and $\boldsymbol{H}_{\mathrm{tan}}$ is the tangential component (amplitude) of the RF part of the magnetic field. For 
an $(n, m)$ Fiske mode in a rectangular junction with length $L$ this reduces to [6]

$$
\begin{aligned}
\Delta P_{\mathrm{s}} \propto & k_{n}^{2} \sin ^{2}\left(k_{n} x_{0}\right) \cos ^{2}\left(k_{m} y_{0}\right) \\
& +k_{m}^{2} \cos ^{2}\left(k_{n} x_{0}\right) \sin ^{2}\left(k_{m} y_{0}\right),
\end{aligned}
$$

where $k_{n}=n \pi / L$, and $k_{m}=m \pi / L$. Here $L, x_{0}$, and $y_{0}$ are the junction length and the beam-position coordinates, respectively. An extension of the model to the multimode case is described in Ref. [8].

In our experiment we obtained LTSEM voltage

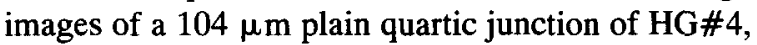

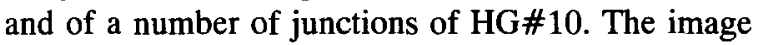
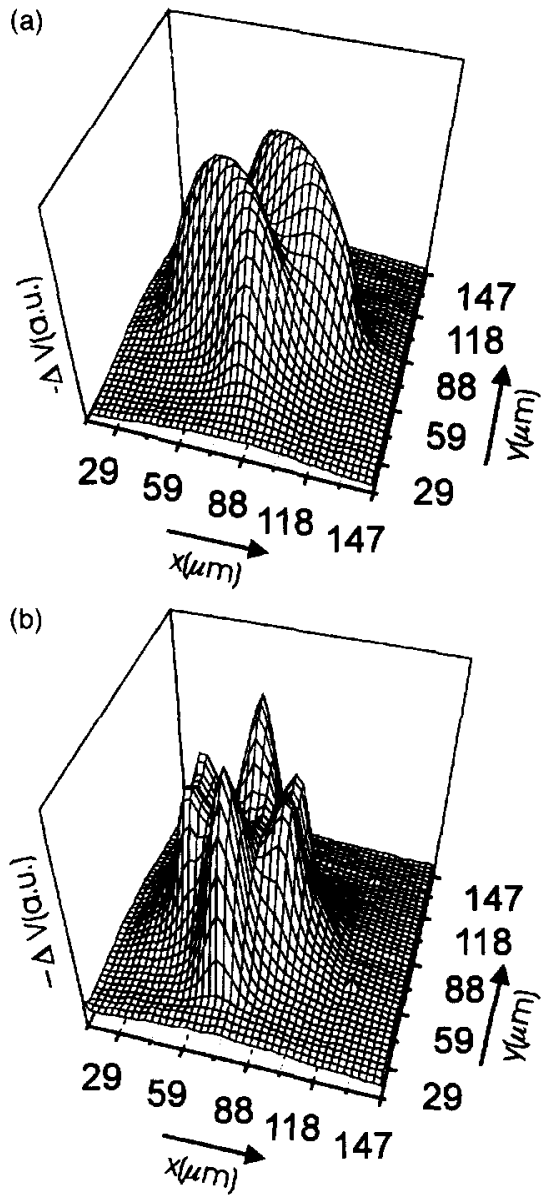

Fig. 7. Three-dimensional LTSEM image of a Fiske resonance at $0.27 \mathrm{mV}$ in a quartic junction of $104 \mu \mathrm{m}$ on HG\#4 (a). The magnetic field $(0.21 \mathrm{mT})$ was applied in the plane of the junction, and at an angle of $154^{\circ}$ with respect to the $x$-axis. Theoretical image of a rectangular $(2,0)$ mode, shown as a wire frame model (b). The $z$-axes display the LTSEM signal in arbitrary units.

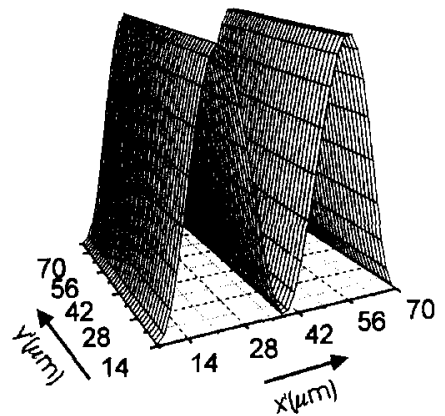

Fig. 8. Three-dimensional LTSEM image of a Fiske resonance at $0.34 \mathrm{mV}$ in a quartic junction of $104 \mu \mathrm{m}$ on HG\#4. The magnetic field $(0.26 \mathrm{mT})$ was applied in the $y$ direction. The $z$-axis displays the LTSEM signal in arbitrary units.

of the resonance found at $0.27 \mathrm{mV}$ for the $104 \mu \mathrm{m}$ plain quartic junction on wafer $\mathrm{HG \# 4}$ is shown in Fig. 7(a). A magnetic field of $0.21 \mathrm{mT}$ was applied at an angle of $154^{\circ}$ with respect to the $x$-axis. In Fig. $7(b)$ the theoretical pattern of a square $(2,0)$ mode at $0.27 \mathrm{mV}$, calculated using Eq. (14), is shown in the $x^{\prime}-y^{\prime}$ coordinate system, where both axes are rotated $45^{\circ}$ with respect to the $x$ - and $y$-axes. The agreement between the modes is clear. For low-order modes, the junction shape does not deviate much from a square in units of resonance wavelengths, and it is justified to call the observed mode a quasi $(2,0)$ mode. Apparently, the magnetic-field direction favors this mode. The observed rounding of the measured pattern at the lower-left side and the top-right side is caused by boundary effects and signal smearing. For most of the resonances at higher voltages it appeared more difficult to obtain stable biasing, especially when the field was applied in the $y$ direction (for which the junction is designed to give a good $I_{c}(B)$ sidelobe suppression). The resonance at 0.34 $\mathrm{mV}$ was quite dominant, however, and its LTSEM image is shown in Fig. 8. The magnetic field ( 0.26 $\mathrm{mT}$ ) was applied in the $y$ direction. All square $(n, m)$ modes that can be calculated by Eq. (14) deviate from the observed pattern, although it remotely resembles a $(2,1)$ or a $(3,1)$ mode. From the LTSEM results we conclude that the higher-order modes do not resemble rectangular modes, which is logical because the deviation of the quartic geometry from the rotated square, in units of resonance wavelengths, is smallest for the low-order modes, for which the best correspondence is expected. 


\section{Conclusions}

It can be theoretically shown, that for small junctions it is possible to use the barrier shape to obtain a very good sidelobe suppression of both the critical current and the Fiske resonances. Junctions with structures inside the barrier area were also considered.

The measurement of the critical current as a function of the magnetic field for a quartic-shaped junction shows an almost perfect sidelobe suppression. The $I_{c}(B)$ curves obtained for the quartic junction with bar, and the quartic junction with a cross inside are modelled with the theory. From the modelling we can conclude that the borders of the internal structures have shifted about $0.75 \mu \mathrm{m}$, and that the self-field effect cannot be completely neglected. A direct fast Fourier transform of the $I_{c}(B)$ data confirmed the results obtained.

As expected from the theoretical argument, the measurement of the Fiske resonances in quarticshaped junctions as a function of the applied magnetic field $I_{\mathrm{F}}(B)$ shows no sidelobes, whereas a lobelike $I_{\mathrm{F}}(B)$ dependence is found for the quarticshaped junction with a bar inside. The sidelobes for the quartic shaped junction with a cross inside are reasonably well suppressed. The initial assumption, that the internal regions are acting as wave breakers, and hence lead to a better suppression, was disproved.

We also obtained images of the resonances using low-temperature scanning electron microscopy. The low-order modes of the quartic junction are quasirectangular, whereas the field patterns of the highorder modes are specific to the exact shape of the junction.

\section{Acknowledgement}

This work in the program of the Foundation for Fundamental Research on Matter (FOM) was partially funded by the Netherlands Technology Foundation.

\section{References}

[1] J.B. le Grand, M.P. Bruijn, M. Frericks, P.A.J. de Korte, E.P. Houwman and J. Flokstra, in: Proc. of the fourth Workshop on Low Temp. Detectors for Neutrinos and Dark Matter (LTD-4), Oxford, 1991 (Editions Frontieres, Dreux, 1992) p. 265.

[2] A.A. Golubov and E.P. Houwman, Physica C 205 (1993) 147.

[3] R.L. Peterson, Cryogenics 31 (1991) 132.

[4] E.P. Houwman, J.G. Gijsbertsen, J. Flokstra and H. Rogalla, Physica C 183 (1991) 339.

[5] J.G. Gijsbertsen, E.P. Houwman, J. Flokstra and H. Rogalla, Physica B 194-196 (1993) 1705.

[6] B. Mayer, T. Doderer, R.P. Hübener and A.V. Ustinov, Phys. Rev. B 44 (1991) 12463.

[7] A. Barone and G. Paternò, Physics and Applications of the Josephson Effect (Wiley, New York, 1982).

[8] S.G. Lachenmann, T. Doderer, R.P. Hübener and D. Quenter, Phys. Rev. B 48 (1993) 3295.

[9] H. Kroger, L.N. Smith and D.W. Jillie, Appl. Phys. Lett. 39 (1981) 280.

[10] R.F. Broom and P. Wolf, Phys. Rev. B 16 (1977) 3100.

[11] J.G. Gijsbertsen, E.P. Houwman, J. Flokstra, H. Rogalla, J.B. le Grand and P.A.J. de Korte, IEEE Trans. Appl. Sup. 3 (1993) 2100

[12] M.A.H. Nerenberg, P.A. Forsyth and J.A. Blackburn, J. Appl. Phys. 47 (1976) 4148.

[13] W.H. Henkels and C.J. Kircher, IEEE Trans. Magn. 13 (1977) 63

[14] R.P. Hübener, in: Advances in Electronics and Electron Physics, Vol. 70 ed. P.W. Hawkes (Academic, New York, $1988)$ p. 1. 